\title{
INFLUENCE OF BEACH LENGTH ON THE DEVELOPMENT OF ESTUARINE DELTAS
}

\author{
Duy Dinh Van, Thuyloi University, dvduy19@gmail.com \\ Hitoshi Tanaka, Tohoku University, hitoshi.tanaka.b7@tohoku.ac.jp \\ Magnus Larson, Lund Universiy, magnus.larson@tvrl.Ith.se \\ Yuta Mitobe, Tohoku Gakuin University, y mitobe@mail.tohoku-gakuin.ac.jp \\ Viet Nguyen Trung, Thuyloi Universiy, nguyentrungviet@tlu.edu.vn
}

\section{INTRODUCTION}

Larson et al. (1987) proposed a closed-form solution to describe the development of river delta coastlines owing to sediment supply from the river. This solution is for a beach of infinite extent adjacent to the river mouth. However, in reality, the beach length is always finite due to the existence of man-made structures or headlands as exemplified in Figure 1. It is, therefore, useful to derive a new analytical solution to gain a better understanding of beach behavior under the effect of boundaries.
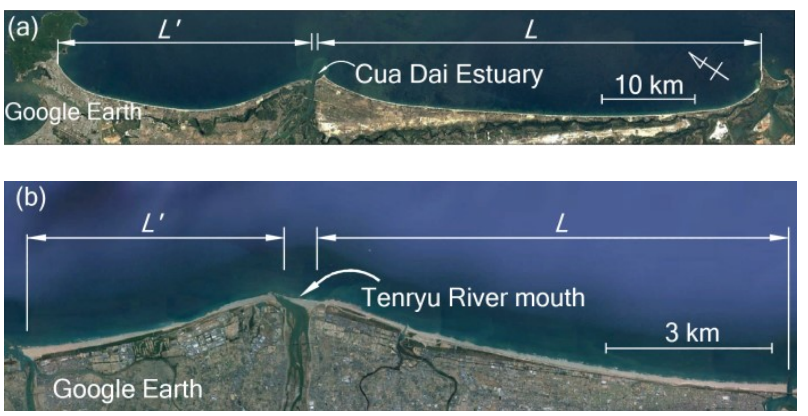

Figure 1 - Examples of beaches with finite lengths ( $L$ and $\left.L^{\prime}\right)$ due to the exitances of headlands in Cua Dai Estuary, Vietnam and man-made structures in Tenryu River delta, Japan.

\section{METHOD}

In this study, a new analytical solution concerning the evolution of the river mouth delta coastlines will be proposed. Characteristic differences between the new analytical solution and the solution provided by Larson et al. (1987) for the formation of river delta shorelines will be discussed. In addition, experimental results of Refaat (1990) will be utilized to validate the new analytical solution.

The new solution with the effect of the boundaries is derived from the simplified governing equation of the one-line model which is identical to the diffusion equation:

$$
\frac{\partial y}{\partial t}=\varepsilon \frac{\partial^{2} y}{\partial x^{2}}
$$

where $t$ is the time and $\varepsilon$ is the diffusion coefficient expressing the time scale of shoreline change, $x$ and $y$ represent the longshore and offshore distances to the shoreline, respectively.

Although there has not been any analytical solution for the development of a river delta coastline with a finite length, there is a comparable study about the heat conduction in a medium between two insulated boundaries (Myers, 1971). It is interesting that restricted sediment input boundaries in the shoreline change model can be considered as a proper analogy to the heat flux and the insulated wall in the heat conduction process. Therefore, the new equation for shoreline evolution under the effect of no-transport boundaries can be obtained as:

$$
y^{*}=\frac{x^{* 2}}{2}-\left|x^{*}\right|+\frac{1}{3}+t^{*}-\frac{2}{\pi^{2}} \sum_{n=1}^{\infty} \frac{e^{-n^{2} \pi^{2} t^{*}}}{n^{2}} \cos \left(n \pi x^{*}\right)
$$

where:

$y^{*}=y\left(2 \varepsilon D / q_{0} L\right), x^{*}=x / L, t^{\star}=\varepsilon t L^{2}$

where $D=D_{\mathrm{B}}+D_{\mathrm{C}}$ is the sum of berm height and depth of closure, $q_{0}$ is the sediment input rate from the river.

Using the above dimensionless expressions, the solution from Larson et al. (1987) can be re-written as:

$$
y^{*}=2 \sqrt{\frac{t^{*}}{\pi}} e^{-\left(x^{* 2} / 4 t^{*}\right)}-\left|x^{*}\right| \operatorname{erfc}\left(\frac{\left|x^{*}\right|}{2 \sqrt{t^{*}}}\right)
$$

where erfc is the complementary error function.

\section{RESULTS}

Figure 2 shows the comparison between Eqs. (2) and (3) for a case similar to Figure 1 with $L=L^{\prime}$. Because the solution is symmetric with respect to the ordinate, it will be displayed on only one side of the symmetry line. As can be seen in Figure 2(a), when the dimensionless time is smaller than 0.1, Eqs. (2) and (3) are in perfect agreement. When $t^{*}$ is around 0.2 , differences start to appear at the right boundary. However, there is no difference at the river mouth $\left(x^{*}=0\right)$. Thereafter, the differences between the two solutions increase and the influence of the boundary becomes clear. From $t^{*}=0.3$, under the effect of the boundary, the shoreline has a parabolic shape that advances parallel in the offshore direction. This can be mathematically explained as $t^{*}$ increases, the fifth term in Eq. (2) becomes zero and the shoreline will advance with a constant speed while keeping its parabolic shape as:

$$
y^{*}=\frac{x^{* 2}}{2}-\left|x^{*}\right|+\frac{1}{3}+t^{*}
$$

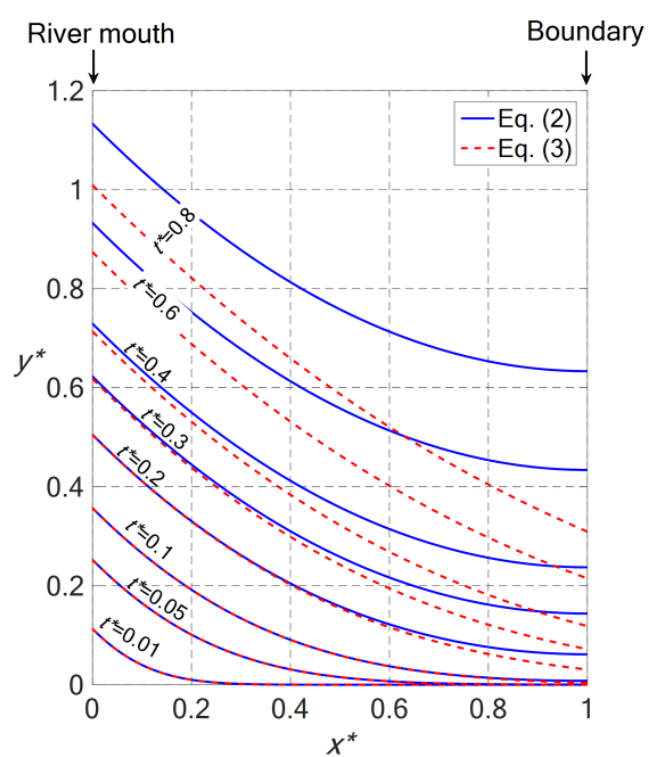

Figure 2. Comparison of shoreline positions between two analytical solution 


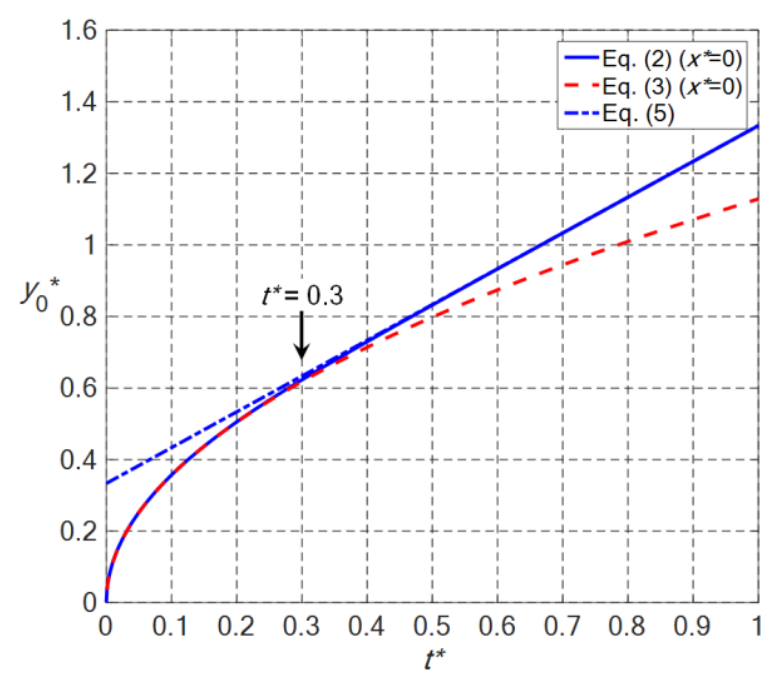

Figure 3. Effect of the boundary on the shoreline evolutions at the river mouth $\left(x^{*}=0\right)$

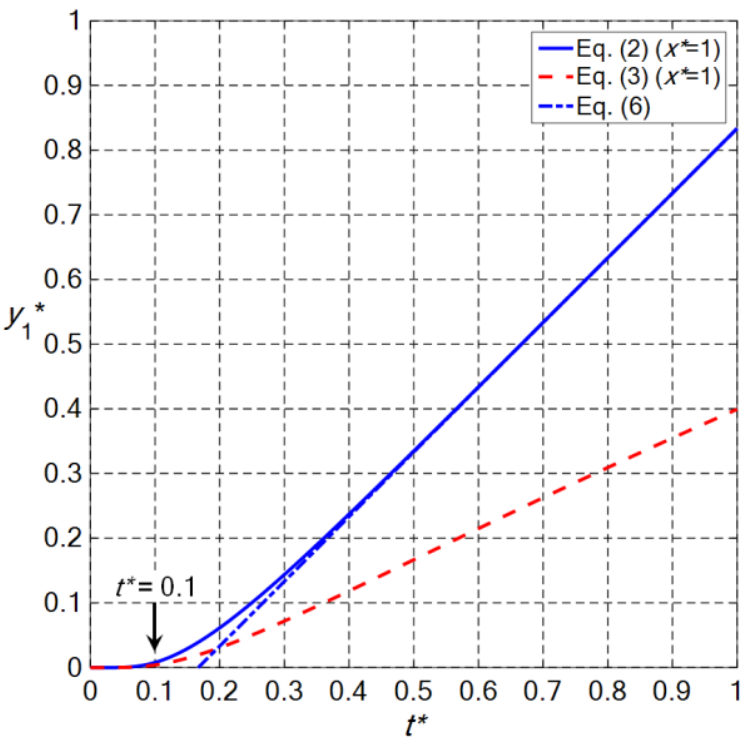

Figure 4. Effect of the boundary on the shoreline evolutions at the lateral boundary $\left(x^{*}=1\right)$

In order to observe the effect of the boundary on the shorelines with respect to time, the shoreline evolutions at the river mouth $\left(x^{*}=0\right)$ and at the boundary $\left(x^{*}=1\right)$ are plotted in Figure 3 and Figure 4.

Figure 3 presents the shoreline evolution at the river mouth $\left(x^{*}=0\right)$. As can be seen from the figure, the difference between two equations occurs around $t^{*}=0.3$ which indicates the effect of the boundary on the shoreline evolution at the river mouth. From the previous discussion in Figure 2, when $t^{*}$ becomes sufficiently large, the shoreline will advance at a constant speed. In Figure 3, this trend is evidenced by the linear segment of the shoreline evolution plotted using Eq. (2). This linear advance of the shoreline with respect to $t^{*}$ can be expressed from Eq. (2) as $\left(x^{*}=0, t^{*} \rightarrow \infty\right)$ :

$$
y_{0}^{*}=t^{*}+\frac{1}{3}
$$

As can be seen in Figure 4 , it is obvious that the difference between two equations happens much earlier at around $t^{\star}=0.1$ at the boundary and the linear portion of the shoreline evolution can also be expressed as:

$$
y_{1}^{*}=t^{*}-\frac{1}{6}
$$

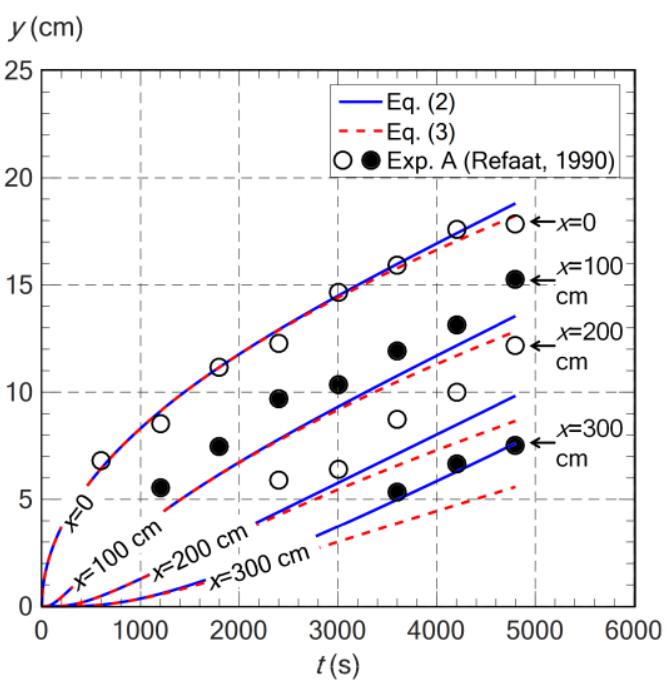

Figure 5 - Comparison with experimental results

In Figure 5, the new solution is validated by making a comparison with experimental results (Refaat, 1990). This experiment was conducted to study the formation process of a river delta in a wave basin which is $8 \mathrm{~m}$ wide. Two waveguide walls are located at about $3.5 \mathrm{~m}$ on both sides of the wave basin. A sediment feeder machine was used to automatically discharge sand as a point source to the wave basin. In the first series of the experiment, sand was discharged constantly at a rate of $7.06 \mathrm{~cm}^{3} / \mathrm{s}$ in 80 minutes. Waves were set to approach normally to the shoreline. The wave height and period are $2.0 \mathrm{~cm}$ and $0.8 \mathrm{sec}$., respectively. Water depth was set constantly at $30 \mathrm{~cm}$. The shoreline positions were recorded at every 10 minutes and $50 \mathrm{~cm}$ interval.

In order to make a comparison, the shoreline evolution was calculated using Eqs. (2) and (3) and the value of $\varepsilon=15 \mathrm{~cm}^{2} / \mathrm{s}$ which is determined based on the shoreline positions recorded from the experiment. The recorded beach profiles in the experiment were also used to obtain the value of $D_{\mathrm{C}}=3.9 \mathrm{~cm}$. As can be seen in Figure 5 , the new solution achieves better agreement with the experimental results, especially near the boundary, which is the side wall of the wave basin $(x=300 \mathrm{~cm})$.

\section{CONCLUSION}

A new analytical solution for the development of river delta coastlines has been obtained. The characteristic differences between the new equation and the equation provided by Larson et al. (1987) for the formation process of river delta shorelines have been discussed. Comparison with experimental results in the case of the no-transport boundary has been made. Good agreement between the new analytical solution and the experimental results shows that the new analytical solution introduced in this study is useful to study the formation process of a river delta with finite shorelines.

\section{REFERENCES}

Larson, Hanson, and Kraus (1987): Analytical solutions of the one-line model of shoreline change, Technical Report CERC-87-15, U.S. Army Engineer Waterways Experiment Station, 72p.

Myers (1971): Analytical Methods in Conduction Heat Transfer, McGraw-Hill, 508p.

Refaat (1990): The formation and reduction process of river delta and their control, Ph.D. Thesis, Kyoto University, $227 p$. 